\title{
A desnutrição proteico-calórica como agravante da saúde de pacientes hospitalizados
}

\author{
Protein-energy malnutrition as an aggravating condition for \\ the health of hospitalized patients
}

Guilherme Malafaia

\begin{abstract}
Resumo
A desnutrição energético-proteica (DEP) é um dos principais problemas de saúde coletiva em escala mundial, por sua magnitude, consequências biológicas e danos sociais. Em pacientes hospitalizados, a DEP é uma realidade altamente prevalente. 0 objetivo desse estudo foi apresentar, por meio de uma revisão bibliográfica, alguns estudos sobre a DEP no âmbito hospitalar; expor os efeitos da desnutrição sobre o sistema imune e, consequentemente, como a DEP pode agravar a saúde do paciente. Por fim, teve como objetivo reforçar a importância do aspecto nutricional para a saúde do paciente hospitalizado. Este estudo apontou para o fato de que a desnutrição hospitalar é um dos principais problemas de saúde pública, tendo como consequências diretas 0 aumento da morbidade e da mortalidade, hospitalização prolongada, e, portanto, aumento dos custos para o sistema de saúde. A desnutrição hospitalar, apesar de ser um problema comum, não é frequentemente identificada como importante na avaliação dos pacientes. Como consequência, a terapia nutricional não é adequadamente prescrita, aumentando, dessa forma, o problema. A DEP pode não apenas afetar adversamente a condição clínica do paciente, como também aumentar seu risco de complicações e, por meio disso, elevar os custos com os serviços de saúde.
\end{abstract}

Palavras-chave: Desnutrição energético-proteica; estado nutricional; hospitalização; saúde; avaliação nutricional.

\begin{abstract}
The protein-energy malnutrition (PEM) is one of the main problems of public health in worldwide scale, for its magnitude, biological consequences and social damages. In hospitalized patients, the PEM is a highly prevalent reality. The objective of this study was to present, through a bibliographic review, some studies on the PEM in the hospital; to expose effects of the PEM on the immune system, consequently, how the PEM can aggravate the health of hospitalized patients and, finally, to strengthen the importance of the nutritional aspect for the health of the patient. The present study reports that the hospital malnutrition is one of the main problems of public health, having as direct consequences the increase of morbidity and mortality, drawn out hospitalization, and, therefore, rise of the costs for the health system. The malnutrition in the hospital, although is a common problem, it is frequently not identified as important in the evaluation of the patients; as consequence, the adequate nutrition therapy is not prescribed, increasing the problem. Zhe PEM can not only affect adversely the clinical condition of the patient, but also increase the risk of complications and costs with the health services.
\end{abstract}

Keywords: Protein-energy malnutrition; nutritional status; hospitalization; health; nutrition assessment.

Recebido: 21/01/2009

Revisado: $24 / 03 / 2009$

Aprovado: 28/04/2009

Programa de Pós-graduação em Ciências Biológicas, Núcleo de Pesquisa em Ciências Biológicas da Universidade Federal de Ouro Preto, Ouro Preto (MG) Endereço para correspondência: Guilherme Malafaia - Rua Vereador Paulo Elias, 8A - Vila Itacolomy - CEP $35400-000$ - Ouro Preto (MG) - E-mail: guilherme@nupeb.ufop.br 


\section{Introdução}

Conforme extensivamente discutido na literatura, a desnutrição energético-proteica (DEP) constitui um dos mais importantes problemas de saúde pública que afeta milhões de pessoas no mundo. A Organização das Nações Unidas para Alimentação e Agricultura (FAO) estimou, em 2004, que 852 milhões de pessoas no mundo apresentavam desnutrição entre os anos de 2000 e 2002, sendo a maioria em países em desenvolvimento ${ }^{1}$. Dados mais recentes da Organização Mundial da Saúde (OMS) ${ }^{2}$ mostram que, nesses países, a desnutrição é responsável, direta ou indiretamente, por $54 \%$ das 10,8 milhões de mortes de crianças com idade inferior a cinco anos.

Particularmente no Brasil, os dados recentes da Pesquisa Nacional de Demografia e Saúde (PNDS), a qual avaliou, dentre outros aspectos, o estado nutricional de crianças com idade inferior a cinco anos indicam uma redução na prevalência da desnutrição infantil no país ${ }^{3}$. Avaliações da prevalência dos déficits de crescimento, em comparações preliminares das PNDS de 1996 e 2006, indicam uma redução de 13 para 7\%. Déficits de peso em relação à altura, indicativos de casos de desnutrição aguda quando sua frequência ultrapassa o limite de 2 a 3\%, foram encontrados em 1,5\% das crianças brasileiras menores de cinco anos, não ultrapassando $2 \%$ em qualquer região ou estrato social da população 3 . Entretanto, mesmo diante dos dados animadores da PNDS 2006, os quais mostram uma tendência à redução da DEP no país, o recente estudo de Coutinho et al. ${ }^{4}$ confirma o alto número de desnutridos que ainda é identificado. Ao realizarem uma análise do perfil nutricional da população brasileira, os autores concluíram que o conjunto de dados disponíveis sobre diferentes grupos etários confirma a alta prevalência da DEP no país, principalmente em regiões mais pobres, como o Norte e o Nordeste.

A alta prevalência da DEP em pacientes hospitalizados foi primeiramente descrita em meados da década de $1970^{5}$, sendo que em ambiente hospitalar, a DEP seria mais bem descrita como processo contínuo que se desencadeia com a ingestão inadequada de nutrientes em relação às suas necessidades e progride por meio de uma sequência de alterações funcionais que precedem as alterações na composição corporal ${ }^{6}$. Schofield \& Ashworth ${ }^{7}$, ao realizar uma revisão de 67 estudos internacionais, mostraram que ao longo de cinco décadas (de 1950 a 1990) o índice de mortalidade de crianças hospitalizadas com DEP grave não mudou, mantendo-se alta (20 a 30\%) e, em alguns locais, atingindo 50 a $60 \%$. A média, nos anos 1990, chegou a 23,5\%7. Enquanto em termos relativos tem-se notado algum progresso nos últimos anos, em termos absolutos o número de desnutridos tem diminuído muito pouco e estudos recentes continuam relatando uma alta incidência de DEP em pacientes hospitalizados, com índices que podem chegar a até $80 \%{ }^{8}$. De acordo com o Ministério da Saúde 9 , no Brasil, a taxa de letalidade hospitalar das crianças com DEP grave internadas é de cerca de $20 \%$, sendo a situação ainda mais preocupante quando se considera que pacientes hospitalizados frequentemente apresentam deterioração do seu estado nutricional ${ }^{9}$.
Estudos apontam para o fato de que a letalidade hospitalar em menores portadores de formas graves de DEP pode chegar a ser dez vezes mais elevada quando comparada com crianças eutróficas ${ }^{10,11}$. Um estudo realizado no Instituto Materno Infantil de Pernambuco (IMIP), por exemplo, observou que, de 1.045 crianças carentes falecidas no hospital no ano de 1995, 60,1\% tinham algum grau de comprometimento do estado nutricional. Em 1998, 15,1\% das crianças desnutridas graves que foram hospitalizadas no IMIP evoluíram para o óbito ${ }^{12}$. Esses dados evidenciam, de forma expressiva, o papel do hospital como centro de demanda dos casos de desnutrição grave.

Assim, este artigo, por meio de uma revisão bibliográfica, tem como objetivo apresentar alguns estudos referentes à DEP no âmbito hospitalar; apresentar de forma específica a forma como a DEP pode afetar o sistema imunológico e, consequentemente, agravar a saúde dos pacientes hospitalizados. Por fim, o artigo objetiva discutir os aspectos relevantes que justificam/reforçam a importância da investigação do estado nutricional de pacientes hospitalizados. Pretende-se explorar essa temática com o intuito de estimular maior atenção dos setores de saúde pública sobre a importância do estado nutricional na clínica médica. Além disso, espera-se que os dados apresentados e discutidos nesse trabalho sirvam de base para a realização de estudos futuros, para intervenções que possam contribuir de modo mais efetivo para melhorar a abordagem médica de pacientes hospitalizados que apresentam DEP.

\section{Método}

Este estudo utilizou materiais de fonte secundária, disponíveis nas Bibliotecas da Universidade Federal de Ouro Preto (UFOP) e no programa de comutação bibliográfica (COMUT), bem como nos bancos de dados Lilacs - Bireme (Base de dados da literatura Latino-Americana e do Caribe de informação em Ciências da Saúde), Medline - Index Medicus (Medical Literature Analysis and Retrieval System Online), SciELO (Scientific Eletronic Library Online) e PubMed (mantido pela National Library of Medicine). Foram selecionados trabalhos que abordaram os principais aspectos sobre a desnutrição no âmbito hospitalar, os efeitos da desnutrição sobre o sistema imune, assim como os estudos que trataram da importância do aspecto nutricional para a saúde do paciente hospitalizado.

A pesquisa bibliográfica incluiu artigos originais, artigos de revisão, comunicação científica, livros, relatórios técnicos, manuais de atendimento do Ministério da Saúde, anais de congresso, teses e dissertações escritas nas línguas inglesa ou portuguesa. Os seguintes termos de pesquisa (palavras-chave e/ou delimitadores) foram utilizados em várias combinações escritos na língua inglesa e/ou portuguesa: "desnutrição", "desnutrição hospitalar", "paciente", "sistema imune”, "saúde", "nutrição hospitalar", "hospitalização", "estado nutricional" e "avaliação nutricional". O recorte temporal abrangeu o período compreendido entre os anos de 1973 e janeiro de 2009, sendo identificados e analisados um total de 48 trabalhos. 


\section{A desnutrição energético-proteica no âmbito hospitalar}

De acordo com Stratton \& Alvarenga ${ }^{13}$, o estudo da DEP no âmbito hospitalar tem sido destaque nos últimos 25 anos. Evidências mostram que a DEP, principalmente quando relacionada a doenças, leva ao aumento da morbimortalidade ${ }^{13}$. Como impacto desfavorável, verifica-se: 1) um comprometimento do estado geral do paciente; 2) custos adicionais para o sistema de saúde e previdenciário; 3) grande ônus social.

A realidade da DEP está documentada em várias publicações científicas em todo mundo. Frutos de pesquisas que se dispuseram estudar a prevalência e o impacto da DEP hospitalar, como por exemplo, os estudos internacionais de Pablo et al. ${ }^{8}$, Singh et al. ${ }^{14} \mathrm{e}$, mais recentemente, Bavelaar et al..$^{15}$. Nesses estudos, aproximadamente $70 \%$ dos pacientes admitidos em hospitais apresentaram DEP após dez dias de internação.

No Brasil, a Sociedade Brasileira de Nutrição Parenteral e Enteral (SBNPE), em 1996, desenvolveu o Inquérito Brasileiro de Avaliação Nutricional Hospitalar (Ibranutri), um estudo epidemiológico transversal, que avaliou o estado nutricional de 4 mil pacientes internados na rede pública de 12 Estados e do Distrito Federal, entre os meses de maio e novembro de $1996^{16}$. Esse estudo revelou que quase metade (48,1\%) dos doentes internados encontrava-se desnutrida. Em 1999, a SBNPE desenvolveu um novo inquérito de avaliação nutricional que teve como objetivo quantificar novamente a desnutrição hospitalar. Os resultados encontrados foram alarmantes: altos índices de desnutrição hospitalar, além da ausência de procedimentos adequados de intervenção que permitiriam uma melhor situação do paciente hospitalizado, referente ao seu estado nutricional ${ }^{17}$.

No estudo epidemiológico transversal de Rezende et al. ${ }^{18}$, os autores, ao avaliarem o estado nutricional de 244 doentes internados em uma instituição filantrópica de Salvador (BA), no mês de março de 2002 , verificaram que mais da metade $(63,11 \%)$ dos pacientes hospitalizados encontravam-se desnutridos. Além disso, observou-se que a desnutrição hospitalar progride à medida que aumenta o período de internação: $46 \%$ dos pacientes ficaram desnutridos nos primeiros cinco dias. Esse número aumentou para $68 \%$ entre 5 e 15 dias e saltou para $83 \%$ nos doentes internados há mais de 15 dias.

Em Alagoas, um dos mais pobres estados da Federação, Ferreira $\&$ França $^{19}$, por meio de um estudo retrospectivo envolvendo 52 crianças, analisaram a evolução do estado nutricional de crianças que estiveram internadas no Hospital Universitário (HU) da Universidade Federal de Alagoas (UFAL). Na ocasião, os autores verificaram que a prevalência de DEP ao final do período de internação foi de $69,2 \%$, apenas dois pontos percentuais abaixo da prevalência inicial. Situação ainda mais preocupante diz respeito à evolução nutricional das crianças internadas especificamente devido à desnutrição. As oito crianças internadas com esse diagnóstico, além de não saírem da categoria de "des- nutrição grave", não apresentaram alteração significativa em termos de evolução em unidades de escore r. Até mesmo o grupo de crianças com diagnóstico de desidratação continuou gravemente desnutrido, apesar do "esperado" incremento de peso obviamente determinado pelo processo de re-hidratação.

Em São Paulo, onde as condições de vida são relativamente melhores que as verificadas em Alagoas, um estudo conduzido na pediatria do Hospital das Clínicas, durante março de 1996 a março de 1997, encontrou prevalência de 55,9\% de DEP entre os pacientes menores de dois anos, e de $35,5 \%$ entre aqueles de dois a cinco anos ${ }^{20}$.

Estes estudos demonstram que a DEP ainda é uma realidade existente no âmbito hospitalar no Brasil, apesar da redução dos índices de DEP no país verificada nos últimos anos. Além disso, reforçam/aumentam a relevância do estudo sobre a desnutrição hospitalar, principalmente quando se analisa os seus efeitos desfavoráveis sobre o prognóstico dos pacientes hospitalizados.

\section{Efeito da desnutrição sobre o sistema imune}

Uma das maneiras com que a DEP agrava a saúde dos indivíduos hospitalizados, a qual será apresentada no presente trabalho, está diretamente associada aos prejuízos imunológicos observados nos pacientes desnutridos, os quais podem apresentar além da desnutrição em si, doenças imunossupressoras e/ou infecto-parasitárias.

Sobre este aspecto, sabe-se que a correlação entre as áreas de nutrição e imunologia, conforme discutido por Field et al. ${ }^{21}$, foi formalmente reconhecida no início da década de 1970 quando medidas da função do sistema imunológico foram introduzidas como componentes de avaliação do estado nutricional de pacientes. Atualmente, tem sido amplamente aceito que a imunidade ou a suscetibilidade a diversas doenças infecto-parasitárias estão diretamente relacionadas ao estado nutricional dos indivíduos.

Segundo Schaible e Kaufmann ${ }^{22}$, a DEP é a causa mais comum de imunodeficiência, usualmente complexa, e que frequentemente envolve não somente proteínas e calorias, mas também variados níveis de deficiências de micronutrientes. Esses nutrientes incluem: fosfato, zinco, cobre, ferro, selênio e as vitamina A, E e as do complexo B, capazes de influenciar diretamente a suscetibilidade a diversas infecções humanas.

Especificamente sobre os prejuízos que a DEP causa no sistema imunológico, estes são múltiplos e conduzem a uma maior suscetibilidade a doenças. Keusch et al. ${ }^{23}$ relataram que crianças desnutridas apresentaram significativa diminuição da função de células $\mathrm{T}$ e um aumento do número de células imunes com falhas no processo de diferenciação. Resultados similares foram relatados por Parent et al..$^{24}$, os quais observaram involução do timo em pacientes infantis desnutridos. Todos os componentes do sistema do complemento - exceto o C4 - estão diminuídos em pacientes desnutridos, particularmente o C3 e o fator $\mathrm{B}^{25,26}$. Tanto a resposta imune celular quanto a resposta imune 
humoral são diretamente afetadas em situações de DEP ${ }^{27,28}$. Em decorrência da involução do timo, que em geral ocorre mais precocemente no quadro de DEP, tanto a estrutura quanto a função deste órgão são prejudicadas e, consequentemente, a resposta de células $\mathrm{T}$ é reduzida ${ }^{29}$. Além disso, outros estudos, conforme revistos por Cunningham-Rundles et al..$^{30}$, demonstraram que a deficiência nutricional afeta particularmente a função fagocítica, produção de anticorpos, citocinas, afinidade do anticorpo pelo antígeno e pelas proteínas do sistema complemento.

No que tange a relação entre a DEP e as doenças infecciosas, em áreas tropicais e subtropicais, estudos importantes têm demonstrado que as deficiências nutricionais podem cronificar e/ou agravar o quadro clínico de algumas doenças, tais como a tuberculose, infecção pelo $\mathrm{HIV}^{31}$ e LV ${ }^{32,33}$. Outras doenças incluem: doenças sexualmente transmissíveis, poliomielite, difteria, tétano, hepatite B e C, hanseníase, dengue, encefalite japonesa e infecções intestinais por nematoides ${ }^{34}$. Além disto, estudos demonstraram que, além de agravar o quadro clínico de diversas doenças infecto-contagiosas, a DEP aumenta as chances de morte prematura em crianças ${ }^{9,35-37}$.

\section{Discussão}

Conforme discutido por Falbo e Alves ${ }^{38}$ e considerada pelo Ministério da Saúde? , a DEP é uma das endemias carenciais mais importantes no Brasil, a qual provoca elevadas demandas nos serviços de saúde e aumento nas taxas de mortalidade hospitalar. Os elevados índices de letalidade verificados em vários estudos (como por exemplo, nos apresentados no presente trabalho), ocorrendo especialmente nas formas graves de DEP envolvendo pacientes hospitalizados, reforçam a importância do estado nutricional para a saúde dos pacientes hospitalizados. Mas quais seriam as razões para a constatação desses elevados índices, apesar da verificação de uma redução na prevalência da DEP em diversas partes do mundo, incluindo o Brasil?

Conforme discutido recentemente por Sarni et al. ${ }^{39}$, talvez uma das causas prováveis para esse fato reside no desconhecimento dos profissionais de saúde com relação à fisiopatologia da DEP grave e/ou da instituição de uma terapia inadequada que resulta em sérias complicações logo nos primeiros dias da internação hospitalar, culminando com a morte. Para Rezende et al. ${ }^{18}$, especificamente no Brasil, existe um grave diagnóstico que, de forma contundente, aflige parcela importante dos profissionais de saúde: sempre houve certo distanciamento das ações voltadas para nutrição por parte da classe médica. Associada ao desconhecimento e descaso da equipe de saúde, a desnutrição dos doentes internados contribui para internações prolongadas e complicações clínicas e diminui a rotatividade dentro dos hospitais, reduzindo ainda mais as vagas disponíveis.

Tratando-se de um estudo que envolva o estado nutricional de pacientes hospitalizados, torna-se inevitável a discussão da atenção dietética nesse contexto, pois é plenamente aceito que uma intervenção dietoterápica bem conduzida é de fundamental importância para um bom prognóstico dos pacientes, sobretudo, se a desnutrição faz parte do quadro nosológico. Segundo Braunschweig et al. ${ }^{40}$, a deterioração do estado nutricional de pacientes hospitalizados, independentemente de sua condição inicial, está associada aos maiores custos hospitalares e maior probabilidade de complicações. Segundo Gallagher-Allred et $a l .^{41}$, o suporte nutricional adequado contribui para redução da prevalência e magnitude da desnutrição, melhora o prognóstico clínico e ajuda a reduzir os custos do tratamento. A investigação do estado nutricional de pacientes hospitalizados está diretamente relacionada com a melhora na recuperação dos pacientes e com menor tempo de hospitalização ${ }^{42}$. Além disso, a falta do diagnóstico nutricional adequado, além de ser prejudicial para a definição do correto tratamento da criança, influencia os dados estatísticos e, portanto, repercute no encaminhamento das políticas e programas para atendimento dos pacientes com desnutrição. No caso da criança com desnutrição grave, a falta do diagnóstico correto dificulta a decisão para o encaminhamento hospitalar oportuno e o adequado tratamento da criança, possibilitando a sua sobrevivência e otimizando sua reabilitação?.

Entretanto, mesmo que estudos tenham evidenciado os benefícios da investigação nutricional do paciente hospitalizado, na prática, o que se tem observado é um quadro diferente. Em muitas ocasiões, a atenção dietética prestada aos pacientes hospitalizados nas clínicas médicas não é efetiva no sentido de melhorar a condição nutricional dos pacientes internados, independentemente do diagnóstico estabelecido inicialmente e do período de hospitalização, conforme verificado no estudo de Ferreira e França ${ }^{19}$, envolvendo crianças.

Há de se ressaltar ainda que, a desnutrição hospitalar não é um problema exclusivo da Pediatria. Pelo contrário, a DEP pode acometer pessoas de todas as faixas etárias, inclusive os mais velhos. Nos idosos, por exemplo, conforme discutido recentemente por Malafaia ${ }^{43}$, os distúrbios nutricionais, como a DEP e a deficiência de micronutrientes, constituem um problema comum, vistos muitas vezes erroneamente, como sendo parte do processo natural do envelhecimento. No estudo de Mühlethaler et al. ${ }^{44}$ realizado na Suíça, com 219 pacientes geriátricos, quase $36 \%$ dos pacientes apresentavam sinais de desnutrição na admissão hospitalar. O estudo revelou que certos sinais de DEP são fortes indicadores de menor sobrevida total e pós-alta nos desnutridos. Em estudo prospectivo de um ano de duração realizado nos Estados Unidos, os pacientes com DEP moderada $(24,4 \%)$ e severa $(16,3 \%)$ apresentaram maior probabilidade de morte ${ }^{45}$.

Diante dos resultados apresentados nesse trabalho, referentes aos estudos que demonstraram os prejuízos da DEP sobre o sistema imunológico, emerge o conceito de "complicações associadas ao estado nutricional”, um dos fatores diretamente associado ao agravo da saúde do indivíduo hospitalizado, o qual passa a apresentar um risco aumentado de morbimortalidade em decorrência do seu estado nutricional. Desta forma, tão importante quanto o diagnóstico de DEP seria a avaliação do risco de deterioração nutricional naqueles pacientes em situações que podem estar associadas a problemas nutricionais ${ }^{46}$. 
Em situações de infecções, por exemplo, o papel da DEP é muito importante na evolução e sobrevivência do indivíduo desnutrido. Conforme argumentado por Pelletier ${ }^{47}$, os efeitos da DEP e da infecção, mesmo nas formas moderadas e leves, não são aditivos, mas sim multiplicativos; fato este que pode agravar ainda mais a saúde do paciente hospitalizado. Além disso, deve-se considerar que a DEP tem sido relacionada ao atraso na capacidade intelectual, baixo rendimento escolar, menor capacidade física para o trabalho, além de ser considerada um determinante do maior risco para gerar crianças com baixo peso ao nascer.

Neste sentido, uma das características das ações para melhorar a nutrição é o seu potencial enquanto instrumento para prevenção e manejo das doenças infecciosas? ${ }^{7}$. O manejo dietético adequado do indivíduo hospitalizado contribui significativamente com a redução da frequência e da gravidade da infecção. Já durante a infecção, o manejo dietético pode modificar o curso e o desfecho da doença, por meio da ingestão adequada de alimentos durante a infecção e a convalescença, particularmente nas crianças pequenas.

Assim, há veemente necessidade de maior atenção por parte dos profissionais ligados à saúde no que se refere ao fator nutricional dos pacientes hospitalizados, principalmente diante do fato de que a avaliação do estado nutricional em indivíduos hospitalizados, muitas vezes, é negligenciada, contribuindo para a ocorrência de complicações e hospitalizações prolongadas. Além disso, conforme demonstrado e discutido no estudo de Rocha et al. ${ }^{48}$, muitas vezes há ocorrência de mudança, para pior, no padrão nutricional de crianças admitidas nos hospitais em condição de eutrofia. Esse fato reforça a importância de uma reflexão sobre a necessidade de uma cultura de valorização do estado nutricional dos pacientes hospitalizados, particularmente crianças e idosos, pelas suas maiores vulnerabilidades nutricionais. $\mathrm{Na}$ ocasião, Rocha et al. ${ }^{48}$ demonstraram que aproximadamente $10 \%$ das crianças eutróficas admitidas em um hospital de Fortaleza, entre agosto e dezembro de 2003, evoluíram para DEP leve e, na alta hospitalar, as crianças admitidas com DEP permaneceram com seu estado nutricional inalterado.

\section{Considerações finais}

Portanto, baseado no que foi exposto, conclui-se que a DEP constitui-se em uma doença altamente importante também no âmbito hospitalar, principalmente quando se considera que o acompanhamento do estado nutricional do paciente hospitalizado culmina em melhoras na sua saúde e em menor tempo de internação.

A desnutrição hospitalar e os impactos negativos deste quadro clínico sobre a saúde dos pacientes são realidades em muitos hospitais, a qual não pode ser negligenciada pelos diversos setores ligados à saúde. Desta forma, é necessário que novos estudos sejam conduzidos com a finalidade de melhor esclarecer o problema, principalmente sobre a sua etiologia no âmbito hospitalar. Além disso, é altamente recomendável que a equipe multidisciplinar responsável pela atenção global dos pacientes hospitalizados discuta os achados referentes à DEP nos hospitais e, se for o caso, reoriente os protocolos de intervenção, dando maior importância ao estado nutricional dos indivíduos internados.

A desnutrição hospitalar desperta interesse não apenas pela alta prevalência e/ou pelo número expressivo de óbitos observados a cada ano, principalmente entre as crianças e os mais idosos, mas, sobretudo, pela natureza do evento. Ao surgir como fator independente de morte, e não como resultado de uma patologia de base (doença renal, cardíaca ou câncer), a desnutrição suscita indagações sobre a etiologia dos quadros carenciais que levam ao óbito. Seria a DEP o resultado de deficiências nutricionais crônicas? Estaria ela relacionada a um quadro carencial de natureza aguda? E/ou à baixa atenção dos profissionais de saúde aos aspectos nutricionais do paciente hospitalizado? Independentemente da fisiopatologia, o problema do pior prognóstico ou dos óbitos por desnutrição em pacientes hospitalizados traz à tona problemas sociais relacionados à desigualdade de acesso e alimentação satisfatória, em quantidade e qualidade. Também traz à tona o papel das políticas públicas.

\section{Referências}

1. Food and Agriculture Organization of the United Nations. FAO. [homepage on the Internet] [cited 2004 Sept]. Available from: http://www.fao.org

2. World Health Organization. Malnutrition: quantifying the health impact at national and local levels. Geneva: World Health Organization; 2005.

3. Brasil. Ministério da Saúde. Pesquisa Nacional de Demografia e Saúde da Criança e da Mulher (PNDS 2006). Ministério da Saúde. Brasília: Ministério da Saúde; 2008

4. Coutinho JG, Gentil PC, Toral N. A desnutrição e obesidade no Brasil: 0 enfrentamento com base na agenda única da nutrição. Cad Saúde Pública 2008;24 (Suppl 2):S332-40.

5. Bistrian BR, Blackburn GL, Vitale J, Cochran D, Naylor J. Prevalence of malnutrition in general medical patients. JAMA 1976;235(15):1567-70.
6. Jeejeebhoy KN. Nutritional assessment. Nutrition 2000;16(7-8):585-90.

7. Schofield C, Ashworth A. Why have mortality rates for severe malnutrition remained so high? Bull World Health Organ 1996;74(2):223-9.

8. Pablo AM, Izaga MA, Alday LA. Assessment of nutritional status on hospital admission: nutritional scores. Eur J Clin Nutr 2003;57(7):824-31.

9. Brasil. Ministério da Saúde. Manual de atendimento da criança com desnutrição grave em nível hospitalar. Brasília: Ministério da Saúde; 2005.

10. McWhirter JP, Pennington CR. Incidence and recognition of malnutrition in hospital. BMJ 1994:308(6934):945-8.

11. Alves JGB, Britto LMA, Melo MAS, Oliveira VA. Morbimortalidade no desnutrido grave hospitalizado. Jornal de Pediatria 1988;64:60-1. 
12. Núcleo de Epidemiologia (NEPI). Boletim Epidemiológico Semestral. Recife: Hospital Geral de Pediatria do Instituto Materno Infantil de Pernambuco; 1998

13. Stratton RJ, Alvarenga A. Combate à desnutrição relacionada à doença: como a intervenção nutricional pode contribuir com benefícios nos resultados clínicos e funcionais, e uma boa relação custo-benefício nos sistemas de assistência à saúde. São Paulo: Support Produtos Nutricionais; 2002.

14. Singh H, Watt $K$, Veitch RM, Cantor M, Duerksen DR. Malnutrition is prevalent in hospitalized medical patients: are housestaff identifying the malnourished patient? Nutrition 2006;22(4):350-4.

15. Bavelaar JW, Otter CD, van Bodegraven AA, Thijs A, van Bokhorst-de van der Schueren MAE. Diagnosis and treatment of (disease-related) inhospital malnutrition: the performance of medical and nursing staff. Clinical Nutr 2008;27(3):431-8.

16. CorreiaMITD.Avaliaçãonutricionalsubjetiva.RBrasNutrClin1998;13(Suppl2): 68-73.

17. Waitzberg DL, Caiaffa WT, Correia MITD. Hospital malnutrition: the Brazilian national survey (IBRANUTRI): a study of 4000 patients. Nutrition 2001;17(7-8): 573-80.

18. Rezende IFB, Oliveira VS, Kuwano EA, Leite APB, Rios I, Dórea YSS, et al. Prevalência da desnutrição hospitalar em pacientes internados em um hospital filantrópico em Salvador (BA), Brasil. R Ci Med Biol 2004;3(2):194-200.

19. Ferreira HS, França AOS. Evolução do estado nutricional de crianças submetidas à internação hospitalar. Jornal de Pediatria 2002;78(6):491-6.

20. Sanabria MC, Dietz E, Varela CA. Evaluación nutricional de niños hospitalizados en un servicio de pediatria de referencia. Pediatr (Asunción) 2000;27(1):16-23.

21. Field CJ, Johnson IR, Schley PD. Nutrients and their role in host resistance to infection. J LeuKoc Biol 2002;71(1):16-32.

22. Schaible UE, Kaufmann SH. Malnutrition and infection: complex mechanisms and global impacts. PLoS Med 2007;4(5):115.

23. Keusch GT, Cruz JR, Torun B, Urrutia JJ, Smith H, Goldstein AL. Immature Circulating Lymphocytes in Severely Malnourished Guatemalan Children. J Pediatr Gastroenterol Nutr 1987;6(2):265-70.

24. Parent G, Chevalier P, Zalles L, Sevilla R, Bustos M, Dhenin JM, et al. In vitro lymphocyte-differentiating effects of thymulin (Zn-Fts) on lymphocyte subpopulations of severely malnourished children. Am J Clin Nutr $1994 ; 60(2): 274-8$

25. Sirisinha S, Edelman R, Suskin DR, Charupatana C, Olson RE. Complement and C3-proactivator levels in children with protein-calorie malnutrition and effect of dietary treatment. Lancet 1973:1(7811):1016-20.

26. Chandra RK. Serum complement and immunoconglutinin in malnutrition. Arch Dis Child 1975;50(3):225-9.

27. McMurray DN. Cellular immune changes in undernourished children. Prog Clin Biol Res 1981:67:305-318.
28. Najera O, Gonzalez C, Toledo G, Lopez L, Ortiz R. Flow cytometry study of lymphocyte subsets in malnourished and well-nourished children with bacterial infections. Clin Diagn Lab Immunol 2004;11(3):577-80.

29. Savino W, Postel-Vinay MC, Smaniotto S, Dardenne M. The thymus gland: a target organ for growth hormone. Scand J Immunol 2002;55(5): 442-52.

30. Cunningham-Rundles S, McNeely D, Ananworanich JM. Immune response in malnutrition. In: Stiehm ER, Ochs HD, Winkelstein JA, editors. Immunologic Disorders in Infants and Children. Philadelphia: Saunders; 2004.

31. Uthman 0. Prevalence and pattern of HIV-related malnutrition among women in sub-Saharan Africa: a meta-analysis of demographic health survey. BMC Public Health 2008;2(8):226.

32. Maciel BLL, Lacerda HG, Queiroz JW, Galvão J, Pontes NN, Dimenstein R, et al. Association of nutritional status with the response to infection with Leishmania chagasi. Am J Trop Med Hyg 2008;79(4):591-8.

33. Malafaia G. Protein-energy malnutrition as a risk factor for visceral leishmaniasis: a review. Parasite Immunol (in press).

34. WHO, Nutrition: Challenges. [database on Internet]. [cited 2005 Jun]. Available from: http://www.who.int/nutrition/challenges

35. Toole MF, Malkki RM. Famine-affected, refugee, and displaced populations: recommendations for public health issues. MMWR Recomm Rep 1992;41(RR-13):1-76.

36. Man WD, Weber M, Palmer A, Schneider G, Wadda R, Jaffar S, et al. Nutritional status of children admitted to hospital with different diseases and its relationship to outcome in The Gambia, West Africa. Trop Med Int Health 1998;3(8):678-86.

37. Buitrón D, Hurtig AK, San Sebastián M. Nutritional status of Naporuna children under five in the Amazon region of Ecuador. Rev Panam Salud Publica 2004;15(3): 151-9.

38. Falbo AR, Alves JGB. Desnutrição grave: alguns aspectos clínicos e epidemiológicos de crianças hospitalizadas no Instituto Materno Infantil de Pernambuco (IMIP), Brasil. Cad Saúde Pública 2002;18(5):1473-7.

39. Sarni RO, Souza FIS, Catherino P, Kochi C, Oliveira FLC, Nóbrega FJ Tratamento da desnutrição em crianças hospitalizadas em São Paulo. Rev Assoc Med Bras 2005;51(2):106-12.

40. Braunschweig C, Gomez S, Sheean PM. Impact of declines in nutritional status on outcomes in adult patients hospitalized for more than 7 days. $J$ Am Diet Assoc 2000; 100(11):1316-22.

41. Gallagher-Allred CR, Voss AC, Finn SC, McCamish MA. Malnutrition and clinical outcomes: the case for medical nutrition therapy. J Am Diet Assoc 1996;96(4):361-6.

42. Correia MITD. Repercussões da desnutrição sobre a morbimortalidade e custos em pacientes hospitalizados no Brasil, São Paulo. Tese (Doutorado) - Faculdade de Medicina da Universidade de São Paulo; 2000.

43. Malafaia G. As conseqüências das deficiências nutricionais, associadas à imunossenescência, na saúde do idoso. Arq Bras Cienc Saude 2008;33(3):168-76 
44. Mühlethaler R, Stuck AE, Minder CE, Frey BM. The prognostic significance of protein-energy malnutrition in geriatric patients. Age Ageing 1995;24(3):193-7.

45. Covinsky KE, Martin GE, Beyth RJ, Justice AC, Sehgal AR, Landefeld CS. The relationship between clinical assessments of nutritional status and adverse outcomes in older hospitalized medical patients. J Am Geriatr Soc 1999;47(5):532-8.
46. McClave SA, Snider HL, Spain DA. Preoperative issues in clinical nutrition. Chest 1999;115(5 Suppl):64S-70S.

47. Pelletier DL. The potentiating effects of malnutrition on child mortality: epidemiologic evidence and policy implications. Nutr Rev 1994;52(12):409-15.

48. Rocha GA, Rocha EJ, Martins CV. The effects of hospitalization on the nutritional status of children. J Pediatr (Rio J) 2006;82(1):70-4. 